MINIAVALIAÇÃO NUTRICIONAL

NA DETERMINAÇÃO DO ESTADO DE SAÚDE

DE IDOSOS COM OU SEM A DOENÇA

DE ALZHEIMER: ASPECTOS POSITIVOS E NEGATIVOS

Patrícia Rocha Castro

Andréa Abdala Frank²

resumo

A população idosa mundial vem crescendo rapidamente, o que representa um desafio para a saúde pública. A longevidade trouxe à tona doenças de natureza crônica, consequentes de processos degenerativos comuns ao envelhecimento. Entre essas demências, a mais prevalente é a Doença de Alzheimer (DA). A DA provoca desordens cognitivas e nutricionais; a perda de apetite e de peso são sintomas que levam a implicações indesejáveis ao prognóstico da doença. Objetivou-se nesta revisão reunir estudos atuais nos quais o questionário de miniavaliação nutricional (MAN) foi utilizado na determinação do estado nutricional de idosos com ou sem a DA, considerando seus aspectos positivos e negativos. As evidências cientificas ressaltam que o questionário da MAN é uma ferramenta simples e rápida na avaliação do estado nutricional de pacientes idosos em clínicas, hospitais e asilos. Estudos revisados indicam que a MAN tem alta especificidade na determinação precoce do risco de desnutrição em idosos, apresentando forte correlação com resultados obtidos através de exames bioquímicos e antropométricos. Percebem-se limitações da MAN quando os idosos avaliados apresentam déficit cognitivo. As dificuldades no preenchimento da MAN devem ser reduzidas com adaptações e esclarecimentos oportunos por parte do pesquisador. Sendo assim, mais estudos devem ser realizados com a aplicação da MAN em idosos demenciados, dada a alta correlação entre estado nutricional com prognóstico da DA.

1 Nutricionista graduada pela Universidade Federal do Rio de Janeiro (UFRJ). Especialista em Nutrição Clínica pelo Instituto de Nutrição Josué de Castro (INJC) da UFRJ. E-mail: patriciarochacastro@hotmail.com

2 Professora Assistente do Departamento de Nutrição e Dietética do INJC/UFRJ. E-mail: andreaabdala@nutrição.ufrj.br 
1 Introdução

A população idosa mundial vem crescendo rapidamente, o que representa um desafio para a saúde pública. No Brasil, têm sido observadas mudanças bruscas no crescimento dessa população, e espera-se que em 2020 este número seja de 32 milhões, comparados com 14 milhões no ano de 2002 (LIMA-COSTA; VERAS, 2003). A DAé a causa mais comum das demências e estima-se que o número de doentes com Alzheimer quadruplique nos próximos 50 anos (GUPTA; LAMONT, 2004).

A expectativa de vida, segundo pesquisa do Instituto Brasileiro de Geografia e Estatística (IBGE) realizada em 2002, era de 71 anos, tendo apresentado um aumento de 4,7 anos em comparação com 1992 (OLIVEIRA et al., 2006). A longevidade da população mundial trouxe à tona doenças de natureza crônica. Dentre essas doenças degenerativas destacam-se as alterações cardiovasculares e as demências, como a Doença de Alzheimer (DA) (WONG; CARVALHO, 2006).

A DA representa a $4^{\mathrm{a}}$ causa de óbito na América do Norte dentre idosos de 75 a 84 anos. Esse tipo de demência provoca a atrofia do córtex temporal mesial (CTM), área do cérebro responsável pelo comportamento alimentar. A DA altera as funções cerebrais concernentes à memória recente, pensamento, linguagem e julgamento crítico (MACHADO et al., 2006).

Perda de memória, desorientação, dificuldades com as atividades da vida diária, inapetência, perda de peso, dificuldades com a fala e a comunicação associam-se fortemente às desordens nutricionais observadas no paciente com DA. A perda de peso leva à redução da massa muscular, da independência e autonomia do doente, aumento da incidência de úlceras de decúbito e infecções sistêmicas, as quais podem agravar o prognóstico da doença (GILLETTEGUYONNET et al., 2000).

$\mathrm{Na}$ fase inicial da doença observa-se perda de peso do idoso, independentemente do consumo alimentar, podendo essa alteração ponderal ser utilizada no diagnóstico (MACHADO et al., 2006).

$\mathrm{Na} \mathrm{DA}$, a perda de peso e a consequente desnutrição ocasionam uma série de complicações que levam à infecção, falência respiratória e insuficiência cardí- 
aca, aumentando a mortalidade. Comparativamente, o ganho ponderal parece ter efeito protetor no desenvolvimento dessa doença (MACHADO et al., 2006).

O tratamento disponível atualmente permite basear a estratégia terapêutica em três pilares: melhorar a cognição, retardar a evolução e tratar os sintomas e as alterações de comportamento. A prioridade do tratamento é identificar e tratar as intercorrências clínicas que possam agravar o estado de saúde geral do idoso (MACHADO et al., 2006).

Suplementos calóricos e nutricionais podem beneficiar a manutenção do peso adequado, ainda que com pequenas perdas ao longo da doença (FRANK; SOARES, 2002).

Contudo, a terapia nutricional somente alcança o objetivo proposto quando associada à avaliação nutricional. Assim, quanto mais precocemente for realizada a avaliação e a intervenção nutricional no paciente com Alzheimer, tanto melhor será o prognóstico de seu quadro. Dessa forma, o seguinte manuscrito objetivou reunir estudos atuais em que o miniquestionário de avaliação nutricional (MAN) foi aplicado em idosos com ou sem a Doença de Alzheimer na determinação do estado nutricional, considerando seus aspectos positivos e negativos.

A metodologia utilizada baseou-se no levantamento bibliográfico dos últimos cinco anos, utilizando-se como base de dados: Periódicos CAPES, SCIELO, LILACS e MEDLINE. Essa revisão foi realizada no período de agosto de 2007 a janeiro de 2008, tendo como palavras-chave: miniavaliação nutricional, estado nutricional, idosos, déficit cognitivo, Doença de Alzheimer.

\section{Doença de Alzheimer}

O termo demência refere-se a sintomas associados a desordens cerebrais com destruição e/ou perda de células nervosas. A velocidade com que a destruição celular acontece caracteriza o quadro clínico de demência em suas diferentes fases (OLIVEIRA et al., 2005).

As demências são doenças que acometem mais os indivíduos idosos, aumentando sua prevalência com o aumento da idade. De 6 a 10\% dos indivíduos com mais de 65 anos sofrem algum tipo de demência. Essa prevalência aumenta de 25 a $48 \%$ nos idosos com idade superior a 80 anos (GUPTA; LAMONT, 2004).

Caracterizada em 1907 por Alois Alzheimer, a DA é de natureza neurodegenerativa, progressiva e irreversível, ocasionando diversos distúrbios de ordem cognitiva. Na maioria dos casos, acomete indivíduos com mais de 60 anos e, quando ocorre em indivíduos mais jovens, denota recorrência fami- 
liar. O crescimento da prevalência de DA é de caráter mundial e associa-se diretamente com o aumento da expectativa de vida, chegando a se colocar como a quarta causa mortis de idosos nos Estados Unidos e Grã Bretanha (SMITH, 1999). Por apresentar grande impacto na qualidade de vida de seus portadores ela é considerada um dos maiores problemas de saúde pública atualmente (PELZER, 2002).

As alterações neurológicas explicam-se pelo acúmulo de proteína $B$-amiloide no cérebro, formando um emaranhado denominado placas amiloides ou placas senis. Essas proteínas normalmente são encontradas sob forma solúvel no fluido cérebro-espinhal e, além delas, parecem ocorrer eventos neuroquímicos ainda não explicados que levam à formação das placas, envolvidas intimamente com a DA e com o gradual declínio das funções cognitivas do indivíduo acometido por essa enfermidade (CHRISTEN, 2000).

Na DA também ocorrem alterações na transmissão de acetilcolina e acetiltransferases. Algumas dessas alterações podem ser encontradas em indivíduos sadios, porém sem causar transtornos cognitivos. Estudos indicam a relação da DA com a presença de genes específicos, e o gene da apolipoproteína $\mathrm{E} 4$ parece contribuir com 50\% de risco para o desenvolvimento dessa demência (SMITH, 1999).

A perda de peso na DA é frequente e ocorre em aproximadamente $40 \%$ dos casos, em todos os estágios, estando presente inclusive antes que seja feito um provável diagnóstico da doença (GUÉRIN et al., 2005).

Uma das explicações para a perda de peso na DA é a hipótese de atrofia do CTM, área do cérebro responsável pelo controle do comportamento alimentar. Sugere-se também que na DA ocorra um aumento das necessidades energéticas que, associado ao baixo consumo alimentar, levaria à redução do peso corporal. A diminuição de peptídeos orexígenos, como o neuropeptídeo Y e a norepinefrina, observada nos portadores da doença, pode levar à perda do apetite, com redução da ingestão alimentar (MACHADO et al., 2006).

A diminuição do peso pode levar à desnutrição, cujo quadro associa-se à piora do prognóstico da DA, aumentando a gravidade das complicações com o surgimento de infecções (GUÉRIN et al., 2005). Segundo Fáxen-Irving, Basun e Cederholm (2005), idosos demenciados apresentam um menor índice de massa corporal (IMC) quando comparados com idosos sem quaisquer alterações cognitivas, e idosas com DA apresentam maior risco de desenvolver desnutrição.

A atrofia do CTM presente na DA pode alternar o quadro de aumento ou não do apetite, além de desequilibrar os processos metabólicos envolvidos na regulação do peso corporal. A agnosia e a apraxia, sintomas decorrentes do 
quadro dessa demência, diminuem a ingestão energética acelerando o processo de perda ponderal, tornando o paciente cada vez mais dependente de seus cuidadores. Outro fator que pode levar à redução do peso é o aumento do requerimento energético em virtude da exacerbada agitação que sofrem os pacientes da DA. Alterações na deglutição, como a disfagia, também acometem indivíduos com Alzheimer, prejudicando a ingestão de alimentos, juntamente com a anorexia causada pela atrofia do CTM (MUÑOZ; AGUDELO; LOPERA, 2006).

Apesar de não haver um consenso no que determina a perda de peso na DA, é fato que ela agrava o prognóstico da doença. Sendo assim, a avaliação nutricional é uma importante e fundamental ferramenta na prevenção da morbimortalidade entre idosos com DA, já que a determinação do estado nutricional do indivíduo permite nortear concretamente o tratamento a seguir, reduzir as complicações da desnutrição e tornar eficaz a terapêutica adotada (RAMOS; VALVERDE, 2005).

Todas as alterações cognitivas decorrentes da DA diminuem a independência do idoso e submetem o mesmo a cuidados especiais. Esses cuidados são geralmente realizados por familiar ou profissional, da área da saúde ou não, na residência do idoso ou em instituições asilares. A figura do cuidador de idosos com Alzheimer representa grande importância na coleta de informações concernentes à rotina desse idoso.

\subsection{Cuidador, família e idosos demenciados}

As demências em geral levam à diminuição da independência do indivíduo devido ao declínio gradual da memória, pensamento, orientação, compreensão, cálculo, capacidade de aprendizagem, linguagem e julgamento (GUERREIRO; CALDAS, 2001).

A assistência ao doente com Alzheimer é dada principalmente pela família, e a figura do cuidador principal é a daquele que responde pela maior parte da atenção prestada ao idoso, principalmente em seu domicílio. Os cuidadores secundários podem ser outros membros da família, voluntários ou profissionais contratados para atuar assistindo o doente. Se o profissional for da área da enfermagem, esse é denominado cuidador formal (CALDAS, 2002).

Idosos demenciados passam por diversas fases de alterações cognitivas que se modificam de acordo com a evolução da doença. Inicialmente, o idoso perde a capacidade de fazer planos, tomar decisões, ter iniciativa e realizar cálculos. A memória apresenta lapsos que podem deixar o idoso desorientado, gerando angústia e depressão, já que esse ainda consegue perceber a 
deterioração de seu estado mental. Conforme a evolução do quadro, necessidades básicas do idoso demenciado passam despercebidas para esses, como a sede e a fome. Muitas vezes esses desidratam e passam um dia inteiro sem se alimentar, sem se darem conta disso (GUERREIRO; CALDAS, 2001).

A não satisfação dessas necessidades básicas, associadas às hipóteses de alterações fisiológicas que podem ocorrer na DA (elevado gasto energético e atrofia do CTM), bem como alterações na aceitação, mastigação, deglutição e assimilação dos alimentos por esses idosos, levam a um rápido e indesejável declínio do estado nutricional (FRANK; SOARES, 2002).

Os cuidados dispensados aos idosos com Alzheimer diferem em muito dos disponibilizados aos idosos não demenciados. Acompanhar um idoso com contínuo declínio cognitivo ocasiona na pessoa do cuidador e na família consequências extremas, que vão da raiva até a frustração (CALDAS, 2002).

Para que a família desenvolva com responsabilidade o processo de cuidar de um indivíduo com Alzheimer, a assistência ao idoso demenciado deve ser considerada questão de saúde pública. O cuidador deve receber apoio de rede social e de saúde que possibilite o melhor atendimento ao seu idoso. A formação de recursos humanos capacitados seria um dos objetivos dessa rede, os quais apoiariam a família no cuidado de seus idosos em seus próprios domicílios, o que diminuiria as internações e possíveis complicações advindas dessa ação (CALDAS, 2002).

O apoio da rede de saúde a nível ambulatorial para o idoso com DA e sua família é muito importante, para que sejam triados aqueles mais suscetíveis a alterações de saúde que possam piorar o prognóstico do Alzheimer. Alguns núcleos de atendimento a idosos realizam a avaliação geriátrica ampla (AGA), em que a avaliação do idoso demenciado ocorre de maneira multidisciplinar, desenvolvendo-se posteriormente um plano de cuidados baseado na identificação de problemas (CALDAS, 2002).

A AGA avalia no idoso com DA a severidade e as implicações de comorbidades presentes, medicações utilizadas e seu impacto sobre o apetite, funcionamento intestinal, continência, cognição, depressão ou sintomas depressivos, mobilidade, dentre outras alterações que, ao serem identificadas precocemente, possam ser melhor enfrentadas pelo idoso (MOTTA, 2001).

Contudo, é o cuidador quem irá ser, após diagnosticada a DA, a janela por onde esse doente irá agora ver o mundo. Será esse cuidador que irá alimentá-lo, vesti-lo e fornecer informações acerca de suas atividades cotidianas, se tornando assim uma pessoa muito importante no tratamento multidisciplinar do idoso, para oferecer as informações relativas ao planejamento de novas terapias ou manutenção das já adotadas. 


\subsection{Avaliação nutricional}

As estatísticas mostram que mais de $85 \%$ dos idosos institucionalizados na Austrália e nos Estados Unidos da América sofrem de desnutrição protéico-calórica, levando ao aumento da morbidade e mortalidade (VISVANATHAN et al., 2003).

A prevalência da desnutrição é grande entre idosos, tanto naqueles institucionalizados quanto nos que vivem em suas residências. Muitas situações afetam o estado nutricional dos idosos, como fatores ambientais, funcionais, psicológicos, nutricionais e médicos. A disfagia, o maior tempo destinado à alimentação, as alterações na mastigação, a perda do apetite e outros aumentam o risco de desnutrição entre os idosos (RODRÍGUEZ et al., 2005).

Idosos desnutridos possuem uma maior chance de adquirir infecções, desenvolver a osteoporose com fraturas ósseas e apresentar problemas cardiorrespiratórios, dentre outras enfermidades que acentuam as desordens nutricionais frequentes com o envelhecimento (CAMPANELLA et al., 2007).

Medidas antropométricas e dados bioquímicos, como proteínas séricas, vêm sendo utilizados na avaliação nutricional de idosos. Entretanto, a prática isolada desses parâmetros parece ter baixa especificidade. Valores antropométricos como IMC, por exemplo, alteram-se em situação de retenção hídrica (edema). Valores bioquímicos como os da albuminemia podem estar alterados em idosos com doenças inflamatórias crônicas, não sendo exclusivo no diagnóstico da desnutrição. A sensibilidade do diagnóstico nutricional parece aumentar quando se utiliza pelo menos um dado antropométrico combinado a um bioquímico (CHRISTENSSON et al., 2002).

Pelo fato da desnutrição em idosos estar comumente ligada às doenças crônico-degenerativas, devem-se levar em consideração critérios clínicos na avaliação do estado nutricional. Nas últimas décadas, vários pesquisadores tentaram encontrar ferramentas confiáveis para avaliar o estado nutricional de idosos e, enquanto não se determina um consenso, muitos métodos diferentes têm sido utilizados (CHRISTENSSON et al., 2002).

O questionário da MAN foi desenvolvido e validado para realizar uma simples e rápida avaliação do estado nutricional de pacientes idosos de clínicas, hospitais e instituições asilares, permitindo a detecção de risco de desnutrição e intervenção nutricional quando necessária (VELLAS et al., 1999). 


\section{Miniavaliação Nutricional Aplicada em ldosos com ou sem a Doença de Alzheimer}

O questionário da MAN é constituído por 18 questões que se dividem em duas partes. Na primeira parte estão contidas seis perguntas, relativas à avaliação da ingestão alimentar e perda ponderal nos últimos três meses, mobilidade, ocorrência de estresse psicológico ou doença aguda recente, alterações neuropsicológicas e IMC. A segunda parte da MAN aborda questões acerca de medidas antropométricas, como circunferências de braço e panturrilha; investigação alimentar, como o número de refeições consumidas, ingestão de líquidos e de alimentos; autonomia para se alimentar; avaliação global, com perguntas relacionadas ao estilo de vida e medicamentos utilizados e uma autoavaliação relativa à saúde e nutrição do idoso (McGEE; JENSEN, 2000).

Para desenvolver e validar a MAN, foi realizado um estudo dividido em três partes. A primeira aconteceu no Hospital Universitário de Toulouse, na França, com a avaliação nutricional de idosos apresentando alterações de saúde, e também com idosos saudáveis da mesma região. Além da aplicação do questionário da MAN, esses idosos tiveram uma avaliação nutricional completa, incluindo os seguintes parâmetros: IMC, circunferência de braço e panturrilha, medida de dobras cutâneas triciptal e subescapular, avaliação bioquímica (albumina, pré-albumina, proteína ligadora de retinol, transferrina, ceruloplasmina, proteína C-reativa, alfa-1-glicoproteína, colesterol, vitaminas A, D, E, B1, B2, B6, B12, folato, cobre e zinco), hemograma completo, inquérito dietético (recordatório alimentar de 3 dias e frequência alimentar) e avaliação do estado geriátrico.

A segunda parte do estudo contou com 120 idosos com alteração de saúde e 30 idosos saudáveis, todos avaliados pela MAN e também por marcadores antropométricos e bioquímicos.

A terceira parte do estudo foi realizada no Novo México, onde a MAN foi aplicada em 347 idosos saudáveis. O objetivo desse estudo foi de validar a utilização da MAN em idosos saudáveis provenientes de um contexto social diferente dos dois primeiros. Os resultados obtidos com a MAN apresentaram alta correlação com os parâmetros nutricionais utilizados. A pesquisa concluiu que a MAN pode ser aplicada em diferentes situações para detectar o risco de desnutrição em idosos.

De acordo com os autores, a aplicação do questionário não necessita de treinamento prévio, e algumas de suas questões não só avaliam o estado nutricional, como ajudam a diagnosticar as causas que podem provocar 
deficiências nutricionais. O que a pesquisa apontou como mais importante na aplicação do questionário foi que o diagnóstico de risco de desnutrição obtido com a MAN pode ocorrer quando o idoso avaliado ainda não apresentar perda ponderal ou alterações séricas de albumina, já que o questionário pode identificar o risco de desnutrição em idosos com baixa ingestão alimentar. Os pacientes que o estudo apontou como em risco de desenvolver desnutrição receberam suporte nutricional e responderam bem a essa intervenção (VELLAS et al., 1999).

Em um estudo transversal, Kuzuya et al. (2005) avaliaram a aplicação da MAN em 226 idosos japoneses com média de idade de 78 anos. Para tanto, foram realizadas medidas antropométricas e marcadores bioquímicos para verificar a validade da MAN como ferramenta de triagem nutricional. De acordo com os resultados obtidos, 19,9\% dos idosos estavam desnutridos, $58 \%$ apresentavam-se em risco de desnutrição e $22,1 \%$ encontravam-se eutróficos. Foram observadas relações significativas entre os resultados da pontuação do questionário com os parâmetros antropométricos e bioquímicos. Os autores concluíram que o questionário da MAN é uma ferramenta efetiva na identificação da desnutrição ou do risco de desenvolver desnutrição em pacientes idosos japoneses.

No estudo de Visvanathan et al. (2003), foi utilizado o questionário da MAN na avaliação do estado nutricional de idosos atendidos pelo serviço de cuidados domiciliares em Adelaide (Austrália). Dos idosos triados para a realização do questionário, foram excluídos os que apresentavam alguma demência, detectada pela aplicação do miniexame do estado mental (MEEM). $\mathrm{O}$ questionário da MAN apresentou alta especificidade e sensibilidade, quando comparado com outros parâmetros de avaliação nutricional, como medidas antropométricas e bioquímicas. Segundo os autores, a MAN é uma ferramenta válida, de fácil aplicação e possibilitou a identificação de idosos desnutridos com maior risco de internação.

O estudo de Persson et al. (2002) avaliou nutricionalmente 83 pacientes do Hospital Universitário de Huddinge, em Estocolmo, na Suécia. Esses pacientes provinham de duas enfermarias do setor de emergência que tratavam os idosos internados com doenças agudas, não incluindo os idosos que apresentavam demência severa. Foram selecionados para o estudo pacientes internados há pelo menos dois dias. Dos 83 pacientes, três apresentavam disfunção cognitiva como motivo principal da internação.

O objetivo da pesquisa foi comparar os resultados obtidos através do questionário da avaliação global subjetiva (AGS) e o da MAN com alguns métodos de avaliação nutricional considerados padrão ouro, tais como: 
dados antropométricos (IMC, circunferência muscular do braço e dobra cutânea triciptal), bioquímicos (albumina sérica) e de composição corporal, como o DEXA (dual energy X-ray absorptiometry ou radioabsorciometria de feixes duplos).

Os resultados mostraram alta correlação entre os valores obtidos através dos questionários da AGS e MAN, e entre esses com os outros parâmetros de avaliação corporal, excluindo os parâmetros bioquímicos. A MAN considerou somente 12 pacientes eutróficos, contra 26 analisados pela AGS. Os idosos identificados como eutróficos pela MAN tiveram uma maior sobrevida do que os identificados pela AGS. Os autores concluíram que a avaliação nutricional no curso de cuidado e tratamento do paciente geriátrico deve incluir o uso concomitante da AGS, MAN e medidas de composição corporal (PERSSON et al., 2002).

Izaola et al. (2005) determinaram o estado nutricional de 145 indivíduos espanhóis hospitalizados, com idade média de 57 anos, utilizando o questionário da MAN, parâmetros bioquímicos e antropométricos. Segundo os itens referentes à MAN, 2,9\% desses indivíduos apresentavam algum tipo de alteração neurológica ou demência. A aplicação da MAN diagnosticou 68,2\% como desnutridos, ao mesmo tempo em que apresentaram valores bioquímicos de albumina, pré-albumina e transferrina abaixo do normal. As medidas antropométricas de circunferência de braço e muscular de braço apresentaram valores abaixo dos recomendados no grupo dos desnutridos. Segundo os pesquisadores, a MAN foi capaz de detectar o risco de desnutrição nos idosos hospitalizados.

Rodriguez et al. (2005) avaliaram 126 idosos venezuelanos, dos quais 70 eram mulheres e 56 homens provenientes de diferentes centros geriátricos, com idade entre 60 a 96 anos. Foram excluídos do estudo indivíduos com deformações físicas, desidratados ou com edema, com enfermidades de natureza catabólica, visceromegalias, alterações cardiovasculares descompensadas, demência e os analfabetos. Os resultados obtidos classificaram como desnutridos 5,6\% dos idosos, 48,4\% como em risco de desenvolver desnutrição e $46 \%$ como eutróficos. De acordo com a faixa etária, os resultados indicaram que idosos com mais de 80 anos apresentaram maior deterioração do estado nutricional do que idosos com idades entre 60 e 79 anos. Os itens do questionário que contribuíram com uma menor pontuação na maior parte dos resultados foram: baixo consumo de líquidos, IMC $<23 \mathrm{Kg} / \mathrm{m}^{2}$ e limitado desempenho físico. A pesquisa apontou que o questionário da MAN deveria apresentar mais questões relacionadas ao índice de atividades de vida diária (AVD) dos idosos. Segundo os autores, o questionário não avalia de- 
talhadamente o desempenho físico do pesquisado. Sabe-se que a diminuição da independência do idoso está diretamente associada à deterioração do estado nutricional. Os pesquisadores também ressaltaram que o questionário apresenta poucas opções de alimentos protéicos para o idoso relatar no item consumo alimentar. $\mathrm{O}$ estudo sugeriu que maiores investigações sejam realizadas na avaliação de fatores de risco para desenvolvimento de desnutrição em idosos venezuelanos.

Ferreira (2005) realizou a avaliação nutricional, através da MAN, com idosos institucionalizados de 60 anos ou mais, na cidade de Guaratinguetá - SP. Foram excluídos da pesquisa os idosos sem possibilidades terapêuticas em fase terminal de doença, além daqueles que não quiseram participar do estudo. Segundo avaliação subjetiva da pesquisadora e de funcionários das instituições asilares, alguns idosos mostraram-se incapazes de responder às questões da MAN devido à presença de distúrbio cognitivo e, nesse caso, os próprios funcionários responderam ao questionário.

O fato dos funcionários terem respondido ao questionário representou uma limitação na aplicação da MAN, já que esses, devido à sobrecarga de trabalho, não puderam fazê-lo de forma fidedigna, pelo fato de não acompanharem todas as atividades na rotina desses idosos. A pesquisadora cita também que algumas questões da MAN não estavam claras quanto ao objetivo proposto. Os pesquisados mostraram-se confusos ao responderem a questão da MAN relacionada à existência de depressão ou demência e, ainda quanto à classificação dessas, se leve ou grave. O estudo também avaliou o grau de sensibilidade das questões relacionadas ao diagnóstico de desnutrição. As questões que apresentaram maior sensibilidade foram: institucionalização, ingestão de líquidos, grau de mobilidade, autopercepção do idoso sobre sua saúde, IMC, circunferência de panturrilha e distúrbios neuropsicológicos. $\mathrm{O}$ diagnóstico nutricional foi de $28,1 \%$ de idosos desnutridos, 50,6\% em risco de desnutrição e 21,3\% de eutróficos (FERREIRA, 2005).

Os resultados do estudo de Ferreira (2005) coincidiram com alguns dos encontrados por Rodríguez et al. (2005), no que diz respeito à sensibilidade das questões da MAN. Nos dois estudos, as questões sobre consumo de líquidos, IMC e mobilidade apresentaram maior sensibilidade para o diagnóstico da desnutrição, já que a maioria dos idosos desnutridos ou em risco de desenvolvê-la apresentou pontuação baixa nessas questões.

Ramos e Valverde (2005) realizaram um estudo transversal no qual se avaliou o estado nutricional de 200 idosos com mais de 85 anos, internados em um hospital em Caracas. Os motivos das internações foram diversos, sendo mais comuns os casos de demência com outras enfermidades associadas, prin- 
cipalmente insuficiência respiratória e cardíaca. A avaliação nutricional foi feita através da aplicação da MAN e de exames bioquímicos; os resultados identificaram $50 \%$ dos idosos como desnutridos e 37,5\% em risco de desenvolver desnutrição. Nesse estudo, foi observada uma correlação estatisticamente significativa entre o risco dos idosos apresentarem desnutrição e sua deterioração psíquica, física e a dependência de cuidadores. O estudo concluiu que a alta prevalência de desnutrição entre idosos hospitalizados foi comprovado tanto com a aplicação da MAN quanto com exames bioquímicos.

O estudo de Matos (2005) avaliou o estado nutricional de 98 idosos provenientes da zona urbana de São Paulo, através da MAN. Também foram aplicados outros questionários elaborados pela pesquisadora, com o objetivo de coletar dados sócio-econômicos que pudessem se correlacionar com a pontuação obtida no MAN, ou seja, identificar quais desses fatores estariam associados a um maior risco de desnutrição. Os resultados indicaram 11,3\% de idosos desnutridos e 37,7\% em risco de desnutrição. A pesquisadora relatou que a MAN foi de fácil aplicação, e que a proporção de idosos em risco de desnutrição foi semelhante à de outros estudos realizados com idosos de comunidades. Em seus resultados, foi identificado que a saúde mental do idoso apresenta forte correlação com a deterioração do seu estado nutricional, conforme também identificado por Ramos et al. (2005). A pesquisa de dados sócio-econômicos indicou que, quanto menor a renda dos idosos (valores inferiores a 2 salários mínimos), pior o prognóstico nutricional.

Wojszel (2006) utilizou a MAN na avaliação nutricional de idosos poloneses institucionalizados. Na identificação do estado geriátrico geral foram utilizados instrumentos como a AVD e escalas de avaliação de demência ou depressão. Segundo a MAN, $12 \%$ dos idosos estavam desnutridos, $61 \%$ estavam em risco de desnutrição e $27 \%$ estavam eutróficos. Tanto os indivíduos diagnosticados como desnutridos quanto os em risco de desnutrição apresentavam suspeita de depressão ou demência, além de problemas com mastigação, pouca mobilidade e elevado número de medicamentos tomados diariamente.

Na pesquisa de Riccio et al. (2007) foi aplicado o MEEM em 47 idosas institucionalizadas, dividindo-as em 3 grupos, de acordo com o grau de demência detectado: leve, moderado ou grave. Os autores utilizaram a MAN para avaliar se o diagnóstico do estado nutricional apresentava alguma correlação com o grau de demência das idosas. Valores bioquímicos para hemoglobina, creatinina sérica e leucócitos também foram obtidos. Identificou-se que, quanto maior a deterioração mental das doentes com Alzheimer, menores as pontuações obtidas na MAN, bem como os níveis séricos de hemoglo- 
bina e leucócitos. Já os valores de creatinina sérica apresentaram-se elevados nas idosas desnutridas.

Andrieu et al. (2001) realizaram um estudo com 318 indivíduos com Doença de Alzheimer recrutados de um hospital especializado nessa demência. Após um ano, 20\% desses pacientes estavam residindo em instituições asilares. Através de análises multivariadas, foram encontrados os seguintes fatores de risco para a institucionalização do idoso: a menor pontuação obtida nesse estudo através da MAN e alterações comportamentais durante as refeições. Para os autores, a aplicação da MAN, além de promover o diagnóstico do estado nutricional, possibilitou uma intervenção nutricional precoce e adequada, podendo prevenir a institucionalização do idoso.

Soini, Routsalo e Lagström (2004) realizaram a avaliação nutricional de 178 idosos finlandeses que recebiam visitas regulares de uma equipe de saúde domiciliar. De acordo com a MAN, 3\% desses idosos foram considerados como desnutridos, sendo que $48 \%$ foram considerados como em risco de desenvolver desnutrição e $49 \%$ foram avaliados como eutróficos. Perda de peso, estresse fisiológico, perda de apetite, autopercepção da saúde e circunferência do braço foram os itens referentes à MAN com maior correlação com o diagnóstico de desnutrição. Os autores consideraram a MAN uma ferramenta útil na identificação de pacientes idosos em risco de desnutrição.

O estudo de Saletti et al. (2005) avaliou o estado nutricional de 353 idosos suecos com idade entre 75 e 89 anos, em atendimento médico domiciliar periódico. A MAN diagnosticou como desnutridos $8 \%$ desses idosos, e $41 \%$ como em risco de desenvolver desnutrição. A perda de apetite foi a questão da MAN que mostrou maior correlação com o diagnóstico de desnutrição.

Pereira (2004) realizou um estudo em que foi avaliado o estado nutricional, através da aplicação da MAN, de 344 idosos no município do Rio de Janeiro. Além da MAN, foram utilizados IMC, circunferência do braço e de panturrilha para avaliar o estado nutricional dos idosos. Houve boa correlação entre os parâmetros antropométricos utilizados e a MAN. Segundo o questionário, $8,3 \%$ os idosos estavam desnutridos, $55,6 \%$ dos idosos se encontravam em risco de desnutrição e $36,1 \%$ estavam eutróficos. A pesquisadora considerou o questionário como uma ferramenta adequada para diagnosticar o risco de desnutrição em idosos.

Emed et al. (2006) avaliaram o estado nutricional de 114 idosos institucionalizados na cidade de Curitiba, através da MAN e do IMC. De acordo com o questionário, $66 \%$ dos idosos avaliados possuíam demência ou depressão, $6 \%$ estavam desnutridos, $61 \%$ encontravam-se em risco de desnutrição e $33 \%$ estavam eutróficos. Segundo o estudo, o questionário da miniavaliação 
nutricional demonstrou detectar com maior precisão o risco de desnutrição do que o valor encontrado pelo IMC. Nesse estudo, a MAN foi importante por apontar grupos vulneráveis à desnutrição que necessitavam de maior investigação clínica.

Os principais objetivos, resultados e conclusões obtidos nos estudos que utilizaram a MAN como ferramenta na avaliação nutricional de idosos com ou sem a doença de Alzheimer, estão descritos em quadro que se encontra no apêndice.

\section{Considerações Finais}

O questionário da MAN foi validado por diversos estudos, em diferentes populações, e o diagnóstico do estado nutricional demonstrou alta correlação com valores bioquímicos e antropométricos considerados padrão-ouro. O elevado custo de alguns exames bioquímicos na avaliação do estado nutricional possibilita que a MAN seja aplicada como medida prioritária, eficaz e de custo reduzido em idosos, objetivando rápida avaliação e intervenção.

Ainda é escasso o número de estudos que utilizam o questionário da miniavaliação nutricional em idosos, sendo ainda menor o conteúdo descrito na literatura com idosos demenciados, especialmente com DA.

Todos os estudos revisados indicaram que a MAN apresenta grande especificidade em detectar precocemente o risco de desnutrição em idosos, o que é de grande valia no acompanhamento de idosos com DA. Nesses casos, o risco de desnutrição é percebido nos primeiros estágios da doença, e correlaciona-se com a piora do prognóstico da mesma.

A aplicação da MAN demonstrou algumas limitações com idosos demenciados. Essas limitações devem-se ao fato de que esses idosos necessitam da participação de seus cuidadores no preenchimento das questões da MAN. No caso de idosos demenciados e institucionalizados, esses cuidadores são funcionários que, muitas vezes, não acompanham com exatidão a rotina dos pacientes devido à sobrecarga de trabalho e a troca rotineira de turnos, inviabilizando a coleta dos dados referentes à avaliação nutricional.

Sabe-se que a desnutrição do idoso, demenciado ou não, pode agravar doenças crônico-degenerativas já existentes, diminuindo sua independência. A avaliação nutricional de idosos deve ser realizada de forma peculiar e única, diferenciada de qualquer faixa etária, utilizando vários outros parâmetros que identifiquem o estado nutricional.

No caso do idoso com a DA, talvez existam maiores dificuldades no preenchimento do questionário da MAN, o que poderia ser minimizado 
com adaptações e esclarecimentos das questões a serem respondidas por cuidadores.

Entende-se que o responsável pela aplicação do questionário deva ser um profissional da área da saúde, com um mínimo de compreensão de cada item a ser abordado, a fim de reduzir o número de dúvidas existentes pelo pesquisado, sem induzir suas respostas.

Dada a alta correlação do estado nutricional com o prognóstico da DA, sugere-se um maior número de estudos utilizando esse instrumento nessa população específica.

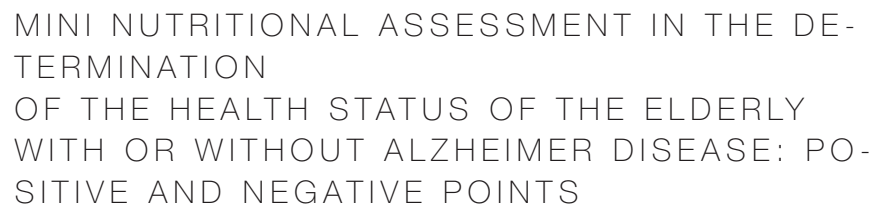

The elderly world population has been growing up quickly, which represents a public health challenge. The longevity brought up chronic diseases, caused by degeneration problems that usually occur in elderly people. Dementias are beyond those diseases, especially the Alzheimer disease (AD). AD causes nutritional and cognitive disorders; low appetite and weight lost are symptoms that lead to undesirable implications to the disease prognostic. This study objected to review actual studies in which the mini nutritional assessment (MNA) was applied in the determination of the nutritional status of elderly, with or without $A D$, considering negative and positive points. Scientific evidences show that MNA questionnaire is a simple and quickly tool on the nutritional assessment of elderly patients from clinics, hospitals and nursing homes. The questionnaire permits the detection of risk of development of malnutrition and nutrition intervention when necessary. The studies reviewed indicate a high specificity of MNA in determining earlier the risk of malnutrition in elderly, and strong correlation between the results obtained from biochemical and anthropometrics exams. Limitations are perceived in MNA when elderly assessed present cognitive deficits. The difficulties in the filling of the MNA must be reduced with adaptations in the questionnaire and with explanations given by the researcher. As so, more studies should be realized with the application of the MNA in elderly demented, given the high correlation between nutritional status and AD evolution. 
keywords

Mini Nutritional Assessment. Nutritional Status. Elderly. Cognitive Deficit. Alzheimer Disease.

\section{referências}

ANDRIEU, Sandrine et al. Nutritional Risk Factors for Institutional Placement in Alzheimer's Disease After One Year Follow-Up. Joumal of Nutrition, Health \& Aging, New York, v. 5, n. 2, p. 113-117, 2001.

CALDAS, Célia Pereira. Contribuindo para a Construção da Rede de Cuidados: trabaIhando com a família do idoso portador de síndrome demencial. Textos sobre Envelhecimento, Rio de Janeiro, v. 4, n. 8, 2002. Disponível em: <http://wnw.unati.uerj.br/tse/ scielo. php? script=sci_arttext\&pid=S1517-59282002000200005\&lng=pt\&nrm=iso $>$. Acesso em: 16 fev. 2008

CAMPANELLA, Luciane Coutinho de Azevedo et al. Relação entre padrão alimentar e estado nutricional de idosos hospitalizados. Revista Brasileira de Nutrição Clínica, São Paulo, v. 22, n. 2, p. 100-108, 2007

CHRISTEN, Yves. Oxidative Stress and Alzheimer Disease. American Joumal of Clinical Nutrition, Bethesda, v. 71, n. 2, p. 621-629, feb. 2000.

CHRISTENSSON, Lennart; UNOSSON, Mitra; EK, Anna-Christina. Evaluation of Nutritional Assessment Techniques in Elderly People Newly Admitted to Municipal Care. European Joumal of Clinical Nutrition, London, v. 56, n. 9, p. 810-818, sept. 2002.

EMED, Thereza Cristina Xavier da Silva; KRONBAUER, Anita; MAGNONI, Daniel. MiniAvaliação Nutricional como Indicador de Diagnóstico em Idosos de Asilos. Revista Brasi leira de Nutrição Clínica, São Paulo, v. 21, n. 3, p. 219-23, jul./set. 2006.

FÁXEN-IRVING, Gerd; BASUN, Hans; CEDERHOLM, Tommy. Nutritional and cognitive relationships and long-term mortality in patients with various dementia disorders. Age and Ageing, London, v. 34, n. 2, p. 136-141, 2005.

FERREIRA, Luciana Silva. Uso da "Mini Avaliação Nutricional" para o Diagnóstico de Des nutrição e Risco de Desnutrição de Idosos Residentes em Instituições de Longa Pemanência. 2005. 197 f. Dissertação (Mestrado em Saúde Pública) - Faculdade de Saúde Pública, Universidade de São Paulo, São Paulo, 2005.

FRANK, Andrea Abdala; SOARES, Eliane de Abreu. Nutrição no Envelhecer. São Paulo: Atheneu, 2002.

GILLEITE-GUYONNET, Sophie et al. Weight Loss in Alzheimer Disease. American Jour nal of Clinical Nutrition, Bethesda, v. 71, n. 2, p. 637-642, feb. 2000.

GUÉRIN, Olivier et al. Different Modes of Weight Loss in Alzheimer Disease: a prospective study of 395 patients. American Joumal of Clinical Nutnition, Bethesda, v. 82, n. 2, p. 435-441, aug. 2005

GUERREIRO, Tânia; CALDAS, Célia Pereira. Memória e Demência: (re)conhecimento e cuidado. Rio de Janeiro: UNATI, UERJ, 2001

GUPTA, Supriya K.; LAMONT, Elizabeth B. Patterns of Presentation, Diagnosis, and Treatment in Older Patients with Colon Cancer and Comorbid Dementia. Joumal of the American Geriatrics Society, New York, v. 52, n. 10, p. 1681-1687, oct. 2004.

IZAOLA, Olatz et al. Mini Nutritional Assessment (MNA). Test as a Tool of Nutritional Evaluation in Hospitalized Patients. Anales de Medicina Interna, Madrid, v. 22, n. 7, p. 313-316, jul. 2005.

KUZUYA, Masafumi et al. Evaluation of Mini-Nutritional Assessment for Japanese Frail Elderly. Nutrition, New York, v. 21, n. 4, p. 498-503, apr. 2005. 
LIMA-COSTA, Maria Fernanda; VERAS, Renato. Saúde Pública e Envelhecimento. Ca demos de Saúde Pública, Rio de Janeiro, v. 19, n. 3, p. 700-701, maio/jun. 2003.

MACHADO, Jaqueline de Sousa; FRANK, Andrea Abdala; SOARES, Eliane de Abreu. Fatores Dietéticos Relacionados à Doença de Alzheimer. Revista Brasileira de Nutrição Clínica, São Paulo, v. 21, n. 3, p. 252-257, jul./set. 2006.

MATOS, Larissa Joana Exposto de Carvalho. Risco de Desnutricão em ldosos na Comunidade. 2005. 97 f. Dissertação (Mestrado em Medicina Social) - Faculdade de Medicina de Ribeirão Preto, Universidade de São Paulo, Ribeirão Preto, 2005.

McGEE, Melissa; JENSEN, Gordon L. Mini Nutritional Assessment (MNA): research and practice in the elderly. American Joumal of Clinical Nutrition, Bethesda, v. 71, n. 1, p. 158, jan. 2000.

MOTTA, Luciana Branco da. Levantamento do Perfil de ldosos intemados em um Hospital Geral: análise do processo de internação frente às demandas da população geriátrica. Textos sobre Envelhecimento, Rio de Janeiro, v. 3, n. 6, 2001. Disponível em: <http://mww.unati.ueri.br/tse/scielo.php?script=sci_arttext\&pid=S1517$59282001000200004 \&$ lng=pt\&nrm=iso >. Acesso em: 16 fev. 2008.

MUÑOZ, Angélica Maria; AGUDELO, Gloria Maria; LOPERA, Francisco Javier. Diagnóstico del Estado Nutricional de los Pacientes con Demencia Tipo Alzheimer Registrados en el Grupo de Neurociencias, Medellín, 2004. Revista Biomédica, Yucatan, v. 26, n. 1 , p. 113-125, marzo 2006.

OLIVEIRA, Maria de Fátima et al. Doença de Alzheimer: perfil neuropsicológico e tratamento. 2005. 21 f. (Trabalho de Licenciatura em Psicologia da Saúde) - Departamento de Psicologia, Universidade Lusíada do Porto, Porto, 2005. Disponível em: <http:// wnw.psicologia.com.pt/artigos/textos/TL0032.PDF>. Acesso em: 20 set. 2007.

OLIVEIRA, Patricia Amante; CUKIER, Celso; MAGNONI, Daniel. Nutrição no Idoso: indicação e discussão de "dietas da moda" em geriatria. Revista Brasileira de Nutrição Clínica, São Paulo, v. 21, n. 1, p. 48-53, jan./mar. 2006.

PELZER, Marlene Teda. A Enfermagem e o Idoso Portador de Demência Tipo Alzheimer: desafios do cuidar no novo milênio. Estudos Interdisciplinares do Envelhecimento, Porto Alegre, v. 4, p. 97-111, 2002.

PEREIRA, Renata Santos. Risco de Desnutrição em ldosos institucionalizados do Município do Rio de Janeiro: um estudo com a Mini Avaliação Nutricional (MAN). 2004. 98 f. Dissertação (Mestrado em Saúde Coletiva) - Instituto de Medicina Social, Universidade do Estado do Rio de Janeiro, Rio de Janeiro, 2004

PERSSON, Margareta D. et al. Nutritional Status Using Mini Nutritional Assessment and Subjective Global Assessment Predict Mortality in Geriatric Patients. Joumal of the American Geriatrics Society, New York, v. 50, n. 12, p. 1996-2002, dec. 2002.

RAMOS, Maria Jesús Gómez; VALVERDE, Francisco Miguel González. Alta Prevalencia de la Desnutrición en Ancianos Españoles Ingresados en un Hospital General y Factores Asociados. Archivos Latinoamericanos de Nutrición. Caracas, v. 55, n. 1, p. 71-76, marzo 2005.

RICCIO, Daniela et al. Comprehensive Geriatric Assessment in Female Elderly Patients With Alzheimer Disease and Other Types of Dementia. Archives of Gerontology and Geriatrics, Amsterdam, v. 44, Supplement 1. p. 343-53, 2007.

RODRÍGUEZ, Nahir et al. Estado Nutricional de Adultos Mayores Institucionalizados Venezolanos. Investigación Clínica, Maracaibo, v. 46, n. 3, p. 219-228, sept. 2005.

SALEITI, Anja et al. Nutritional status and a 3-year follow-up in elderly receiving support at home. Gerontology, Basel, v. 51, n. 3, p. 192-198, may/june 2005

SMITH, Marilia de Arruda Cardoso. Doença de Alzheimer. Revista Brasileira de Psiquiatria, São Paulo, v. 21, p. Sll3-SII7. Suplemento 2, out. 1999. 
SOINI. Helena: ROUTASALO, Pirkko: LAGSTRÖM, Hanna. Characteristics of the Mini Nutritional Assessment in Elderly Home-Care Patients. European Journal of Clinical Nutri tion, London, v. 58, n. 1, p. 64-70, jan. 2004.

VELLAS, Bruno et al. The Mini Nutritional Assessment (MNA) and its Use in Grading the Nutritional State of Elderly Patients. Nutrition, Burbank, v. 15, n. 2, p. 116-122, feb. 1999.

VISVANATHAN, Renuka et al. The Nutritional Status of 250 Older Australian Recipients of Domiciliary Care Services and its Association with Outcomes at 12 Months. Joumal of the American Geriatrics Society, New York, v. 51, n. 7, p. 1007-1011, July 2003.

WOJSZEL, Zita Beata. Determinants of Nutritional Status of Folder People in Long-Term Care Settings on the Example of the Nursing Home in Bialystok. Advances in Medical Sciences, Bialymstoku, v. 51, p. 168-173, 2006.

WONG, Laura L. Rodriguez; CARVALHO, José Alberto. O Rápido Processo de EnveIhecimento Populacional do Brasil: sérios desafios para as políticas públicas. Revista Brasileira de Estudos de População, São Paulo, v. 23, n. 1, p. 5-26, jan./jun. 2006.

\section{APÊEDICE}

Resultados de estudos que utilizaram a MAN na avaliação nutricional de idosos.

\begin{tabular}{|c|c|c|c|c|}
\hline & Estudo & Objetivo & Resultados & Conclusão \\
\hline 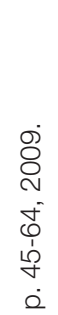 & $\begin{array}{l}\text { Vellas } \\
\text { et al. } \\
\text { (1999) }\end{array}$ & $\begin{array}{c}\text { Desenvolvimento } \\
\text { e validação da MAN } \\
\text { através da aplicação } \\
\text { do questionário em grupos } \\
\text { de idosos fragilizados, } \\
\text { saudáveis, institucio- } \\
\text { nalizados ou não, } \\
\text { provenientes de diferentes } \\
\text { contextos sociais. }\end{array}$ & $\begin{array}{l}\text { Os autores observaram } \\
\text { que a MAN apresenta } \\
\text { alta correlação com parâ- } \\
\text { metros antropométricos e } \\
\text { bioquímicos. }\end{array}$ & $\begin{array}{l}\text { A MAN detecta o risco } \\
\text { de desnutrição } \\
\text { em diferentes grupos } \\
\text { de idosos quando ainda } \\
\text { não estão presentes } \\
\text { alterações ponderais } \\
\text { e dos níveis } \\
\text { de albumina sérica. }\end{array}$ \\
\hline $\begin{array}{l}\dot{0} \\
\dot{0} \\
0\end{array}$ & $\begin{array}{c}\text { Kuzuya } \\
\text { etal. } \\
\text { (2005) }\end{array}$ & $\begin{array}{l}\text { Avaliação nutricional de } \\
226 \text { japoneses idosos } \\
\text { utilizando a MAN, parâ- } \\
\text { metros antropométricos e } \\
\text { bioquímicos. }\end{array}$ & $\begin{array}{l}\text { O estudo observou } \\
\text { relações significativas } \\
\text { entre o questionário } \\
\text { e parâmetros antropo- } \\
\text { métricos e bioquímicos. }\end{array}$ & $\begin{array}{l}\text { A MAN identificou o risco } \\
\text { de desenvolver desnutri- } \\
\text { ção e apresenta } \\
\text { correlação significativa } \\
\text { com valores antropo- } \\
\text { métricos e bioquímicos. }\end{array}$ \\
\hline $\begin{array}{l}\circ \\
\stackrel{0}{0} \\
0\end{array}$ & $\begin{array}{c}\text { Visvanathan } \\
\text { et al. } \\
\text { (2003) }\end{array}$ & $\begin{array}{c}\text { Avaliação nutricional } \\
\text { de idosos sem demência, } \\
\text { atendidos pelo serviço } \\
\text { de cuidados domiciliares } \\
\text { na Austrália. }\end{array}$ & $\begin{array}{l}\text { A MAN apresentou alta } \\
\text { especificidade e sensibili- } \\
\text { dade quando comparada } \\
\text { com medidas antropo- } \\
\text { métricas e bioquímicas. }\end{array}$ & $\begin{array}{l}\text { A MAN identificou } \\
\text { que idosos em risco } \\
\text { de desnutrição } \\
\text { apresentam maiores índi- } \\
\text { ces de internação. }\end{array}$ \\
\hline
\end{tabular}




\begin{tabular}{|c|c|c|c|}
\hline $\begin{array}{l}\text { Persson } \\
\text { et al. } \\
(2002)\end{array}$ & $\begin{array}{l}\text { Avaliação nutricional de } 83 \\
\text { pacientes sem demência } \\
\text { internados em um hospital } \\
\text { na Suécia, utilizando } \\
\text { a MAN e a ASG. }\end{array}$ & $\begin{array}{c}\text { Idosos identificados } \\
\text { pela MAN como eutróficos } \\
\text { tiveram maior sobrevida } \\
\text { dos que identificados } \\
\text { pela ASG. }\end{array}$ & $\begin{array}{l}\text { Os autores concluíram que } \\
\text { a avaliação nutricional do } \\
\text { idoso deve incluir } \\
\text { a aplicação da MAN, ASG } \\
\text { e medidas de composição } \\
\text { corporal. }\end{array}$ \\
\hline $\begin{array}{l}\text { Izaola } \\
\text { et al. } \\
(2005)\end{array}$ & $\begin{array}{c}\text { Avaliação nutricional de } \\
\text { idosos espanhóis hospita- } \\
\text { lizados. }\end{array}$ & $\begin{array}{l}\text { Idosos identificados como } \\
\text { desnutridos pela MAN tive- } \\
\text { ram valores bioquímicos } \\
\text { e antropométricos abaixo } \\
\text { dos padrões. }\end{array}$ & $\begin{array}{c}\text { A MAN é capaz } \\
\text { de identificar risco } \\
\text { de desnutrição } \\
\text { em idosos hospitalizados. }\end{array}$ \\
\hline $\begin{array}{l}\text { Rodriguéz } \\
\text { et al. } \\
\text { (2005) }\end{array}$ & $\begin{array}{c}\text { Avaliação nutricional } \\
\text { de idosos venezuelanos } \\
\text { sem demência } \\
\text { provenientes de centros } \\
\text { geriátricos. }\end{array}$ & $\begin{array}{c}\text { As questões da MAN } \\
\text { que contribuíram com uma } \\
\text { menor pontuação } \\
\text { foram relativas à baixa } \\
\text { ingestão de líquidos, } \\
\text { IMC<23 kg/m² e limitado } \\
\text { desempenho físico. }\end{array}$ & $\begin{array}{l}\text { A MAN deveria } \\
\text { apresentar mais questões } \\
\text { relativas à AVD. }\end{array}$ \\
\hline $\begin{array}{c}\text { Ferreira } \\
(2005)\end{array}$ & $\begin{array}{c}\text { Avaliação nutricional com } \\
\text { idosos institucionalizados } \\
\text { da cidade } \\
\text { de Guaratinguetá, } \\
\text { São Paulo. }\end{array}$ & $\begin{array}{l}\text { As questões da MAN } \\
\text { com maior sensibilidade } \\
\text { ao diagnóstico } \\
\text { de desnutrição foram } \\
\text { relativas ao grau de mobili- } \\
\text { dade, IMC, circunferência } \\
\text { de panturrilha, ingestão } \\
\text { de líquidos e distúrbios } \\
\text { neuropsicológicos. }\end{array}$ & $\begin{array}{l}\text { A MAN apresenta limi- } \\
\text { tações quanto à clareza } \\
\text { de algumas questões, } \\
\text { principalmente quando } \\
\text { um cuidador responde } \\
\text { pelo idoso que apresenta } \\
\text { algum distúrbio cognitivo } \\
\text { que o impossibilite de } \\
\text { preencher o questionário. }\end{array}$ \\
\hline $\begin{array}{l}\text { Ramos } \\
\text { et al. } \\
(2005)\end{array}$ & $\begin{array}{l}\text { Avaliação nutricional } \\
\text { de idosos hospitalizados } \\
\text { em Caracas, Venezuela. }\end{array}$ & $\begin{array}{c}\text { Observou-se correlação } \\
\text { significativa entre risco de } \\
\text { desnutrição e deterioração } \\
\text { psíquica e física. }\end{array}$ & $\begin{array}{l}\text { A MAN e valores bio- } \\
\text { químicos identificaram } \\
\text { índices de desnutrição em } \\
\text { grande parte dos idosos. }\end{array}$ \\
\hline $\begin{array}{l}\text { Matos } \\
(2005)\end{array}$ & $\begin{array}{l}\text { Avaliação nutricional de } \\
\text { idosos da zona urbana de } \\
\text { São Paulo. }\end{array}$ & $\begin{array}{l}\text { A saúde mental do idoso } \\
\text { apresentou forte correlação } \\
\text { com a deterioração de seu } \\
\text { estado nutricional. }\end{array}$ & $\begin{array}{c}\text { A pesquisadora conside- } \\
\text { rou o questionário de fácil } \\
\text { aplicação. }\end{array}$ \\
\hline $\begin{array}{c}\text { Wojszel } \\
\text { (2006) }\end{array}$ & $\begin{array}{c}\text { Avaliação nutricional de } \\
\text { idosos poloneses institu- } \\
\text { cionalizados. }\end{array}$ & $\begin{array}{l}\text { Segundo a MAN, a maioria } \\
\text { dos idosos estava em } \\
\text { risco de desnutrição. }\end{array}$ & $\begin{array}{l}\text { Idosos diagnosticados } \\
\text { como desnutridos } \\
\text { apresentavam suspeita } \\
\text { de depressão ou demência, } \\
\text { além de apresentarem } \\
\text { dificuldades de mobilidade, } \\
\text { mastigação e ingerirem } \\
\text { diariamente elevado } \\
\text { número de medicamentos. }\end{array}$ \\
\hline
\end{tabular}




\begin{tabular}{|c|c|c|c|c|}
\hline 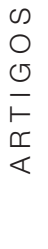 & $\begin{array}{l}\text { Riccio } \\
\text { et al. } \\
(2007)\end{array}$ & $\begin{array}{l}\text { Avaliação de idosas } \\
\text { institucionalizadas } \\
\text { com diversos graus } \\
\text { de demência. }\end{array}$ & $\begin{array}{c}\text { Idosas identificadas } \\
\text { como desnutridas pela } \\
\text { MAN e com maior grau } \\
\text { de demência, segundo } \\
\text { o MEEM, apresentaram } \\
\text { valores bioquímicos abaixo } \\
\text { dos padrões. }\end{array}$ & $\begin{array}{l}\text { Quanto maior a deterio- } \\
\text { ração mental das idosas, } \\
\text { menores as pontuações } \\
\text { obtidas com a MAN. }\end{array}$ \\
\hline & $\begin{array}{l}\text { Andrieu } \\
\text { et al. } \\
\text { (2001) }\end{array}$ & $\begin{array}{l}\text { Avaliação nutricional } \\
\text { de idosos com DA. }\end{array}$ & $\begin{array}{c}\text { Idosos com menor } \\
\text { pontuação na MAN tive- } \\
\text { ram um índice } \\
\text { de internação } 20 \% \text { maior } \\
\text { que os demais um ano } \\
\text { após a avaliação. }\end{array}$ & $\begin{array}{l}\text { A MAN promove } \\
\text { o diagnóstico nutricional } \\
\text { e possibilita intervenção } \\
\text { que pode prevenir institu- } \\
\text { cionalização do idoso. }\end{array}$ \\
\hline & $\begin{array}{l}\text { Soini } \\
\text { et al. } \\
\text { (2004) }\end{array}$ & $\begin{array}{l}\text { Avaliação nutricional } \\
\text { de idosos finlandeses } \\
\text { atendidos por equipe } \\
\text { de saúde domiciliar. }\end{array}$ & $\begin{array}{c}\text { As questões da MAN } \\
\text { com maior correlação } \\
\text { com o diagnóstico } \\
\text { de desnutrição foram: } \\
\text { perda de peso, estresse } \\
\text { fisiológico, perda } \\
\text { de apetite, autopercepção } \\
\text { da saúde e circunferência } \\
\text { do braço. }\end{array}$ & $\begin{array}{l}\text { A MAN é uma ferramenta } \\
\text { útil na identificação } \\
\text { de pacientes idosos } \\
\text { em risco de desnutrição. }\end{array}$ \\
\hline & $\begin{array}{l}\text { Saletti } \\
\text { et al. } \\
(2005)\end{array}$ & $\begin{array}{l}\text { Avaliação nutricional } \\
\text { de idosos suecos com } \\
\text { atendimento domiciliar } \\
\text { periódico. }\end{array}$ & $\begin{array}{c}\text { A questão da MAN } \\
\text { com maior correlação com } \\
\text { diagnóstico de desnutrição } \\
\text { foi a da perda de apetite. }\end{array}$ & $\begin{array}{l}\text { A MAN é ferramenta útil } \\
\text { no diagnóstico } \\
\text { da desnutrição. }\end{array}$ \\
\hline 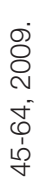 & $\begin{array}{l}\text { Pereira } \\
(2004)\end{array}$ & $\begin{array}{c}\text { Avaliação nutricional } \\
\text { de idosos do município } \\
\text { do RJ. }\end{array}$ & $\begin{array}{l}\text { Houve boa correlação en- } \\
\text { tre os resultados obtidos } \\
\text { com a MAN e parâmetros } \\
\text { antropométricos utilizados. }\end{array}$ & $\begin{array}{c}\text { A pesquisa aponta que } \\
\text { a MAN é uma ferramenta } \\
\text { adequada no diagnóstico } \\
\text { do risco de desnutrição } \\
\text { em idosos. }\end{array}$ \\
\hline $\begin{array}{l}\dot{2} \\
\dot{5} \\
\dot{\check{C}} \\
\dot{5} \\
\dot{\square}\end{array}$ & $\begin{array}{l}\text { Emed } \\
\text { et al. } \\
\text { (2006) }\end{array}$ & $\begin{array}{l}\text { Avaliação nutricional de } \\
\text { idosos institucionalizados } \\
\text { da cidade de Curitiba utili- } \\
\text { zando a MAN e o IMC. }\end{array}$ & $\begin{array}{l}\text { A MAN demonstrou de- } \\
\text { tectar com maior precisão } \\
\text { o risco de desnutrição do } \\
\text { que os valores de IMC. }\end{array}$ & $\begin{array}{l}\text { A MAN foi considerada } \\
\text { importante por apontar gru- } \\
\text { pos vulneráveis à desnu- } \\
\text { trição e que necessitem } \\
\text { maior investigação clínica. }\end{array}$ \\
\hline
\end{tabular}

Recebido: 25-08-2008

1a Revisão: 28-11-2008

2a Revisão: 07-04-2009

Aceite Final: 13-04-2009 